\title{
Stéréotypes de genre et programmes cachés d'éducation dans l'enseignement en France. Une revue de littérature sur les inégalités occultées
}

\author{
Jacques Gleyse \\ Université de Montpellier, Montpellier, France
}

\begin{abstract}
Résumé
L'article proposé est constitué d'une revue de littérature générale et quelques études spécifiques et originales sur la question de l'égalité filles-garçons à l'école en France. II propose une délimitation théorique des concepts de genre et de sexe au travers notamment des rôles sexués. Ensuite, après un parcours historique assez rapide, il montre comment la mixité mise en place en 1976 dans tous les établissements scolaires français, ne correspond pas du tout à l'égalité des chances ni à l'égalité de traitement des élèves filles et des élèves garçons. Les préjugés et stéréotypes de genre reste très nombreux dans les écoles de ce pays. L'article en montre de manière résumé certaines facettes à différents niveaux de la scolarité et dans la plupart des matières mais aussi au travers de question par exemple, de la transgresion des règles scolaires et des punitions.
\end{abstract}

Mots-clés

Genre. Egalité. Filles-garçons. Ecole. Enseignement.

\section{Estereótipos de gênero e programas educacionais ocultos em educação na} França. Uma revisão da literatura sobre desigualdades ocultas

\begin{abstract}
Resumo
O artigo apresenta uma revisão bibliográfica ampla e alguns estudos especializados e originais sobre o tema da igualdade de gênero nas escolas francesas. Inicialmente, propõe-se uma delimitação teórica dos conceitos de gênero e sexo através dos papéis de gênero. Em seguida, após uma breve análise histórica, mostra-se como o sistema de coeducação introduzido em 1976 em todas as escolas francesas não corresponde à igualdade de oportunidades ou de tratamento para meninas e meninos. Os preconceitos e estereótipos de gênero ainda estão muito presentes. De forma sintética, apontam-se facetas desses estereótipos nos diferentes níveis de escolaridade e na maioria das disciplinas, explorando inclusive questões como a transgressão das regras escolares e a punição.
\end{abstract}

Palavras-chave

Gênero. Igualdade. Menina-Menino. Escola. Ensino. 


\title{
Gender stereotypes and hidden education programs in education in France.
}

\section{A literature review on hidden inequalities}

\begin{abstract}
The proposed article consists of a review of general literature and some specific and original studies on the issue of gender equality at school in France. It proposes a theoretical delimitation of the concepts of gender and sex, in particular through gender roles. Then, after a fairly rapid historical overview, it shows how the co-educational system introduced in 1976 in all French schools does not correspond at all to equal opportunities or equal treatment of girls and boys. Gender prejudices and stereotypes are still very numerous in schools in this country. The article summarizes some facets of these prejudices and stereotypes at different levels of schooling and in most subjects, but also through issues such as the transgression of school rules and punishments.
\end{abstract}

\section{Keywords}

Gender. Equality. Girl-boy. School. Education.

\section{Introduction}

La mixité dans l'école est acquise depuis 1976 et les décrets d'application de la loi Haby. Pour autant, cette mixité ne signifie pas nécessairement l'égalité de traitement et de considération des deux sexes.

S'il y a une quarantaine d'années, les garçons dominaient largement l'espace et les positions scolaires, aujourd'hui la situation s'est sensiblement modifiée puisqu'environ 70000 filles de plus que de garçons obtiennent le baccalauréat et que celles-ci, tendent à devenir meilleures que les garçons dans la plupart des disciplines scolaires. Toutefois lles enquêtes P.I.S.A. (voir, entre autres : enquête P.I.S.A., 2018 ${ }^{1}$ ) à 15 ans montrent que les filles ont un an d'avance sur les garçons en lecture, et que cet écart tend à s'accroître, mais qu'à l'inverse les garçons ont 3 mois d'avance en mathématiques et que cet écart ne se réduit que très lentement. Par contre, toutes les enquêtes depuis leur création (2000) montrent que les filles sont davantage « scolaires » que les garçons. La mixité dans tous les cas n'est donc pas l'égalité de réussite.

Cependant, si les filles réussissent de mieux en mieux à l'école et les garçons de moins en moins bien, les positions sociales qui en résultent à l'âge adulte sont quasiment inversées. A la sortie de l'école les salaires des femmes sont de 16,7\% inférieurs aux salaires des hommes à plein temps (et $31 \%$ tous temps de travail confondu, et donc $25 \%$

1 https://www.oecd.org/pisa/pisa2018\%20_resumés_i-ii-iii.pdf

Educ. Form., Fortaleza, v. 6, n. 2, e4625, maio/ago. 2021

DOI: https://doi.org/10.25053/redufor.v6i2.4625

https://revistas.uece.br/index.php/redufor/index ISSN: 2448-3583 
en équivalent temps plein) ${ }^{2}$ et surtout un " plafond de verre » (BUSCATTO; MARRY, 2009) existe qui empêche les femmes d'accéder aux positions les plus prestigieuses dans la société, par exemple dans les conseils d'administrations des plus grandes entreprises privées ou publiques ou même dans les instances dirigeantes des administrations publiques (par exemple dans les universités scientifiques ou médicales) et, bien sûr, dans la sphère politique. II serait sans doute possible d'attribuer cela à des inclinations naturelles. Mais les travaux de sciences humaines et sociales et de sciences de l'éducation conduisent à une toute autre conclusion: les facteurs culturels sont prédominants pour expliquer les inégalités de traitement. Cela est démontré depuis plusieurs décennies en France (parmi d'autres : BELOTTI, 1974 ; BOURDIEU, 1999 ; DURU-BELLAT, 1990 ; MOSCONI, 1989). Au Brésil et al. (CAMPONAR et al., 2020 ; SOUZA RIOS ; DE MELO CARDOSO ; FERREIRA DIAS, 2018 ; RODRIGUEZ, 2020) de nombreux travaux existent également de longue date mais ce n'est pas l'objet de cet article.

On peut donc imaginer que, si des éléments socioculturels jouent un rôle dans ce processus, l'école peut probablement participer, tout autant que l'éducation parentale, à la mise en place d'une inégalité entre les filles et les garçons, inégalité qui doit certainement être une inégalité de traitement. C'est cela que l'on dénomme hide curriculum et que l'on peut traduire par "programme caché d'éducation », nous y reviendrons plus loin.

\section{Le genre et le sexe : approche épistémologique et méthodologique}

II faut immédiatement expliquer, avant d'aborder la question du genre à l'école, que le sexe est naturel et biologique alors que le genre est social, culturel et historicisé. Ces deux éléments ont, évidemment des conséquences en termes de comportement et de psychologie individuelle.

En tout état de cause, on naît mâle ou femelle. Cette dichotomie est partagée par de très nombreuses espèces animales. Autrement dit, pour les êtres humains nous sommes dotés d'une vingt-troisième paire de chromosomes XX (femelle) ou XY (mâle)

2 Voir à ce sujet: https://www.inegalites.fr/Les-inegalites-de-salaires-entre-les-femmes-et-les-hommes-etatdes-lieux

Educ. Form., Fortaleza, v. 6, n. 2, e4625, maio/ago. 2021

DOI: https://doi.org/10.25053/redufor.v6i2.4625

https://revistas.uece.br/index.php/redufor/index 
qui généralement déterminent le sexe d'un individu. Les femelles sont dotées de vagin, utérus, trompes, ovaires, vulve et clitoris, les mâles de testicules et de pénis. II existe toutefois un tout petit nombre de personnes intersexuées (environ une sur un million en France) dont on ne peut clairement identifier le sexe à la naissance soit en raison d'un brouillage des signes sexuels apparents, soit en raison d'une non adéquation des organes sexuels et du patrimoine génétique.

Le genre lui, correspond au féminin et au masculin. II est différent selon les sociétés et les époques. II est évidemment culturel. II existe en psychologie sociale des échelles de masculinité et de féminité qui, si elles ont été longtemps opposées ou inversement dynamiques semblent de plus en plus considérées comme cumulatives (Bem Sex Role Inventory, BEM, 1974). Autrement dit, on peut être très féminin et très masculin sur une même échelle ou peu féminin et peu masculin. Ce qui signifie que ces composantes ne sont plus considérées comme exclusives ou antagonistes. En général, il est possible cependant d'identifier socialement ce que l'on appelle des stéréotypes sociaux de genre. Williams John E. \& Susan M. Bennett dans un article paru en 1975 dans Sex Roles: "The definition of sex stereotypes via the adjective check list» (WILLIAMS ; BENNETT, 1975) ont listé les qualificatifs les plus fréquemment attribués aux hommes et aux femmes avec une significativité pertinente bien que réalisée sur un échantillon restreint. Les deux tableaux ci-dessous décrivent ces stéréotypes :

Tableaux 1 - Stéréotypes pour les hommes

\begin{tabular}{|c|c|c|}
\hline Affirmatif & Désordonné & Indépendant \\
\hline Agressif & Dominant & Inexcitable \\
\hline Ambitieux & Élégant & Logique \\
\hline Autocratique & Endurant & Masculin \\
\hline Aventureux & Énergique & Rationnel \\
\hline Bruyant & Enjoué & Réaliste \\
\hline Casse-cou & Entreprenant & Rigoureux \\
\hline Confiant & Excitable & Robuste \\
\hline Constant & Ferme & Sans-émotion \\
\hline Courageux & Fort & Sévère \\
\hline Cruel & Grossier & Vantard \\
\hline
\end{tabular}

Source : Williams et Bennett (1975). 
Tableaux 2 - Stéréotypes pour les femmes

\begin{tabular}{|c|c|c|}
\hline Affectueuse & Douce & Pleurnicheuse \\
\hline Attentive & Élogieuse & Préoccupée \\
\hline Attirante & Emotionnelle & Prudente \\
\hline Capricieuse & Excitable & Rêveuse \\
\hline Charmante & Faible & Sentimentale \\
\hline Charmeuse & Féminine & Sensible \\
\hline Complaisante & Frivole & Sophistiquée \\
\hline Cœur tendre & Humble & Soumise \\
\hline Délicate & Nerveuse & Volubile \\
\hline Dépendante & Persévérante & Volage \\
\hline
\end{tabular}

Source : Williams et Bennett (1975).

Cette même structuration stéréotypée est décrite, toujours au travers d'adjectifs attributifs, dans un article, plus tardif, cherchant à établir une vision globale du phénomène puisqu'il est question, là, de pan-cultural Gender Stereotypes. Le tableau qui, pourtant, ne touche pas un seul pays, comme précédemment, mais 25 fait apparaître des stéréotypes relativement similaires. Voici le document extrait de l'article de Williams, Satterwhite \& Best (1999) :

Table I. Pancultural Gender Stereotypes: Samples of Hightly Stereotypic Items ${ }^{a}$

\begin{tabular}{|c|c|c|c|c|c|}
\hline \multicolumn{3}{|c|}{ Male Stereotype } & \multicolumn{3}{|c|}{ Female Stereotype } \\
\hline Item No. & Adjective & $\mathrm{M} \%$ & Item No. & Adjective & $\mathrm{M} \%$ \\
\hline 2 & Active & 81 & 5 & Affected & 20 \\
\hline 4 & Adventurous & 93 & 6 & Affectionate & 10 \\
\hline 7 & A ggre ssive & 88 & 11 & Anxious & 23 \\
\hline 10 & Ambitious & 82 & 18 & Attractive & 14 \\
\hline 19 & A utocratic & 86 & 30 & Charming & 19 \\
\hline 35 & Coarse & 91 & 38 & Complaining & 21 \\
\hline 50 & Courage ous & 86 & 53 & Curious & 24 \\
\hline 52 & Cruel & 79 & 61 & Dependent & 19 \\
\hline 53 & Daring & 86 & 71 & Dreamy & 17 \\
\hline 70 & Dominant & 87 & 77 & Emotional & 12 \\
\hline 78 & Energetic & 82 & 83 & Fearful & 17 \\
\hline 79 & Enterprising & 81 & 86 & Feminine & 8 \\
\hline 90 & Forceful & 93 & 98 & Fussy & 24 \\
\hline 122 & Independent & 84 & 149 & Meek & 25 \\
\hline 136 & Inventive & 81 & 151 & Mild & 22 \\
\hline 143 & Logical & 79 & 220 & Sensitive & 14 \\
\hline 147 & Masculine & 96 & 224 & Sexy & 14 \\
\hline 186 & Progressive & 78 & 230 & Shy & 25 \\
\hline 209 & Robust & 85 & 240 & Soft-Hearted & 19 \\
\hline 210 & Rude & 83 & 253 & Submissive & 16 \\
\hline 213 & Self-Confident & 79 & 256 & Superstitious & 13 \\
\hline 248 & Stern & 84 & 261 & Talkative & 22 \\
\hline 251 & Strong & 92 & 268 & Timid & 25 \\
\hline 271 & Tough & 91 & 293 & Weak & 17 \\
\hline 279 & Unemotional & 82 & 294 & Whiny & 23 \\
\hline
\end{tabular}

Source : Williams, Satterwhite et Best (1999).

Educ. Form., Fortaleza, v. 6, n. 2, e4625, maio/ago. 2021

DOI: https://doi.org/10.25053/redufor.v6i2.4625

https://revistas.uece.br/index.php/redufor/index 
On peut constater que les stéréotypes sexués sont assez proches de ceux qui avaient été mis en évidence 24 ans plus tôt. Autrement dit, cela signifierait que les rôles masculins et féminins n'évoluent que très lentement dans le temps. Toutefois lorsque l'on observe les changements des apparences vestimentaires, par exemple, ou de coiffure on constate des modifications importantes entre 1970 et aujourd'hui, en Occident et en Orient. Pour l'Occident alors que la féminité était associée aux cheveux courts et à une apparence androgyne, aujourd'hui elle est davantage associée à des cheveux longs et à des formes suggestives. Pour la masculinité il en va de même si les cheveux longs étaient l'apanage des rock-stars des années 70 aujourd'hui au contraire les cheveux courts dominent avec une légère barbe.

On pourrait réaliser la même analyse pour le Moyen-Orient et l'Orient mais avec d'autres déclinaisons davantage fondées sur des croyances religieuses ou des mutations sociopolitiques. Les stéréotypes seraient donc labiles et modifiables.

Une étude a été réalisée par nos soins portant sur 850 étudiants de master 1 se destinant à tous les métiers d'enseignement secondaire et d'encadrement (62\% de femmes et 38\% d'hommes : l'enseignement secondaire est de plus en plus féminisé). II a été demandé de fournir les 5 qualificatifs qui correspondaient le mieux à une fille ou à un garçon de Gème (ce niveau de classe a été choisi car la composante sexuée y est moins apparente). On retrouve parmi les 15 qualificatifs significativement les plus cités :

Tableaux 3 - Les qualifications citées dans l'étude

\begin{tabular}{|c|c|}
\hline Filles & Garçons \\
\hline Appliquées & Agités \\
\hline Bavardes & Bagarreurs \\
\hline Emotives & Sportifs \\
\hline En binômes & Non scolaires \\
\hline «Fashion victims » & Directs \\
\hline Font des coups en douce & En groupes \\
\hline Littéraires & Testent les limites \\
\hline Matures & Jeux vidéos \\
\hline « Pestes » & Scientifiques \\
\hline Pipelettes & Provocateurs \\
\hline Propres & Sales \\
\hline Sages & Conflictuels \\
\hline Scolaires & Bruyants \\
\hline Studieuses & Immatures \\
\hline Sérieuses & Compétitifs \\
\hline Source : Prepare par l'auteur
\end{tabular}

Source : Préparé par l'auteur. 
De manière lapidaire, il est possible d'affirmer que généralement les filles se situent dans le « care », le souci de l'autre et les garçons davantage dans l'agir, que les filles sont globalement décrites comme scolaires et les garçons comme non scolaires. La position stéréotypale du care se retrouve aujourd'hui dans le fait que les métiers du soin (y compris les études médicales où l'on retrouve $56,3 \%$ de filles) et de l'éducation, voire de l'aide juridique (avocats et droit 65\% de femmes) sont généralement féminisés et les métiers technologiques et industriels généralement masculinisés. En tout état de cause, il reste à savoir si l'éducation parentale et surtout, du point de vue qui le notre, si l'éducation scolaire diminue ou accroît les stéréotypes de genre, si elle en est l'un des vecteurs ou non.

\section{L'égalité et la différence : la nécessité d'une distinction théorique}

L'égalité est un des principes fondateurs de la République française. Liberté, Egalité, Fraternité figurent sur le fronton des mairies qui séparaient d'ailleurs souvent, au début du siècle, une école de garçons et de filles, dans les hameaux ou très petits villages. Mais le concept d'égalité en soi ne veut rien dire. II s'agit généralement de l'égalité en droits, devoirs et dignité (« les hommes naissent et demeurent libres et égaux en droit »). Mais pour ce qui est de l'école et d'un certain nombre d'autres structures il faudrait y ajouter l'égalité de traitement. Traite-t-on les filles et les garçons de manière égale dans leur éducation en général et dans l'école en particulier ? Cela est loin d'être une évidence comme le montre Jacques Gleyse (2020).

II convient de préciser, sur un autre plan, qu'égalité s'oppose à inégalité et non, selon une erreur fréquemment commise sur ce sujet, à différence. On peut être différents et égaux en termes de traitement, de droits, de devoirs et de dignité.

A la différence s'oppose l'uniformité ou la similitude pas l'égalité. Or la question que se pose le système scolaire depuis quelques temps maintenant puisque les premières actions officielles ont commencé autour des années 2000 en France, c'est bien celle de l'inégalité de traitement des élèves dans un contexte de mixité relativement général. Or les stéréotypes de genre tout autant que certains programmes cachés d'éducation véhiculés dans l'école pourraient être à l'origine de la mise en place d'inégalités de traitement mais aussi par voie de conséquence d'inégalités en droits, devoirs et dignité des filles et des garçons. $Y$ compris dans des domaines tels que la 
punition ou l'interaction, on va y revenir plus loin, les filles et les garçons ne sont pas traités à la même aune.

\section{L'histoire de l'école : des filles longtemps absentes de l'enseignement secondaire}

II n'est pas possible dans l'espace d'un article de développer longuement cette question mais il convient de constater que les filles ont - selon l'expression d'Antoine Prost - longtemps été élevées "sur les genoux de l'église » (PROST, 1992), étant en cela discriminées dans leurs apprentissages. Et même si les salons bourgeois et aristocratiques, autour de la Révolution française associent des intellectuelles comme Olympe de Gouge, Anne-Catherine Helvétius, Sophie de Grouchy à Voltaire ou Condorcet, les collèges et plus tard les lycées sont réservés aux garçons. C'est seulement en 1882 grâce aux lois Jules Ferry et Camille Sée que l'école primaire devient laïque, mixte et obligatoire pour les deux sexes, mais en 1888 encore Jules Simon, ministre de l'Instruction publique des beaux-arts et des cultes considère que "seul un esprit d'homme mûr peut étudier la chimie pratique » (cité par : PROST, 1992, p. 45).

En réalité, des classes à plusieurs niveaux, pour l'enseignement primaire, dans des hameaux ou de tout petits villages où n'est nommé qu'un seul instituteur ou qu'une seule institutrice, sont mixtes par obligation (on connaît l'exemple, datant de 1938, de la classe de Rogues, décrite par Adrienne Durand-Tullou, dans : Le Pays des asphodèles ou encore des classes de Célestin Freinet à Bar-sur-Loup à partir de 1928). Dans la même logique, les Cours Complémentaires, pour l'enseignement primaire supérieur associant généralement les meilleurs élèves d'une école de filles et d'une école de garçons, sont mixtes.

Mais dans l'enseignement secondaire et dans les Ecoles Primaires Supérieures et Ecoles Normales d'instituteurs, la situation est tout autre. II existe des lycées de jeunes filles, généralement littéraires et des lycées de garçons, généralement scientifiques hermétiquement clos à l'autre sexe et le baccalauréat, lui même, est différent pour les deux sexes jusqu'en 1924. Les programmes pour les filles et les garçons ne seront pas identiques pendant tout le début du XXe siècle.

Sous des contraintes économiques, la gémination se fait jour à partir de 1957 (les garçons littéraires vont dans les lycées de filles et les filles scientifiques dans les lycées de garçons). En 1959, la mixité devient légale dans les lycées et il en va de même des collèges 
à partir de la réforme Fouchet-Capelle, en 1963. Enfin, comme on l'a vu, ce sont les décrets d'application de la loi Haby qui instaurent la mixité obligatoire. Toutefois encore aujourd'hui dans les lycées professionnels ou techniques, de nombreuses sections sont démixées du fait des options prises par les élèves. La mixité n'existe donc véritablement que depuis une quarantaine d'année dans le système secondaire français (une discipline comme l'Education Physique y a résisté jusqu'aux années 90). Mais la mixité signifie-t-elle pour autant l'égalité de traitement des filles et des garçons et l'égalité en droits, devoirs, dignité ?

Aujourd'hui les filles réussissent bien mieux à l'école, à peu près dans toutes les disciplines, cependant elles obtiennent un bac littéraire à $70 \%$ et les garçons un bac scientifique ou technique à $60 \%$. Dans les classes préparatoires les filles sont présentes à $74 \%$ dans les filières littéraires et seulement à $30 \%$ dans les filières scientifiques. Quant ils se jugent très bon en mathématiques huit garçons sur dix choisissent une filière scientifique. Dans la même situation ce sont seulement six filles sur dix qui font ce choix. II convient donc de s'interroger également sur les déterminants, peut-être scolaires, de ces choix.

Depuis 2000 deux conventions interministérielles pour l'égalité entre les filles et les garçons, les femmes et les hommes dans le système éducatif, ont tenté de mettre en œuvre une politique d'égalité commune à plusieurs ministères. En 2013 seront mis en œuvre ce que l'on a appelé les $A B C D$ de l'égalité et qui sont simplement des fiches de séquences scolaires réalisées par des enseignants ou des enseignants-chercheurs visant à rétablir davantage d'égalité de traitement entre les filles et les garçons à l'école. Enfin le code de l'éducation affirme : « Les écoles, les collèges, les lycées [...] contribuent à favoriser la mixité et l'égalité entre les hommes et les femmes, notamment en matière d'orientation. Ils assurent une formation à la connaissance et au respect des droits de la personne ainsi qu'à la compréhension des situations concrètes qui y portent atteinte ».

Cet article 121-1 du code de l'éducation reprend l'article 5 de la loi d'orientation et de programme pour l'avenir de l'École du 23 avril 2005.

\section{Les programmes cachés d'éducation sur le genre : une découverte de la fin du XXe siècle}

En 1973, dans l'environnement féministe qui suit et précède les évènements de 1968, l'ouvrage d'Elena Gianini Belotti, Du côté des petites filles, qui, d'une certaine 
manière, bien que n'appartenant pas au même courant féministe, prolonge le travail de Simone de Beauvoir dans Le Deuxième sexe (1949) mais le limitant à l'éducation familiale, fait l'effet d'une bombe dans un monde où la naturalisation du genre reste une évidence massivement partagée. L'ethnologue des familles italiennes démontre là que dès la sortie du ventre maternel une fille n'est pas traitée de la même manière qu'un garçon et que ces comportements répétés fabriquent en quelque sorte une « faible femme » et un garçon «fort ». L'ensemble des actes, des gestes, des paroles prononcées et des techniques utilisées montre que la culture familiale opère un traitement très différencié des petites filles et des petits garçons. Cela suggère que ce traitement différencié n'est pas du tout issu de fondements naturels mais bien culturels dans une société patriarcale. Ces traitements pourraient aujourd'hui être considérés comme des «programmes cachés d'éducation », dans la mesure où ils ne sont jamais explicités et explicites. Cet ouvrage permet, en tout état de cause, de commencer à réfléchir sur l'idée de genre et de dissocier la question du genre de celle du sexe dans le processus éducatif même si les mots ne sont pas aussi explicites alors.

Dès les années soixante-dix les Feminist studies (revue crée en 1970) ont bien pointé l'inégalité de représentation qui existe dans les manuels scolaires de lecture et même de science ou d'histoire géographie. En règle générale les hommes sont toujours présentés de manière dominante et les femmes de manière dominées. Souvent les femmes sont assignées aux tâches domestiques et les hommes aux travaux dits «nobles». Des patterns de comportement sont aussi décrits qui montrent les garçons actifs et les filles souvent passives mais aussi cantonnées à l'usage de poupées, au ménage, au repassage, etc. (GLEYSE, 2020). D’autres travaux, dans cette lignée (BOURDIEU, 1999), montreront que les attentes des parents sont très différentes pour une fille que pour un garçon et certains montrent encore récemment que des parents qui attendent un premier enfant prioritairement et majoritairement souhaitent avoir un garçon.

Mais il y a plus puisque lorsque l'on étudie les manuels d'histoire on constate que seuls les hommes y ont une place centrale et que les femmes ont disparu, en grande partie de la geste historienne, à quelques exceptions notables comme Jehanne d'Arc, Aliénor d'Aquitaine, Catherine de Médicis et Louise Michel, voire, plus récemment Marie Curie. Souvent on découvre le rôle des femmes lorsqu'elles « remplacent » les hommes

Educ. Form., Fortaleza, v. 6, n. 2, e4625, maio/ago. 2021 
dans les usines (les femmes travaillent dans l'industrie textile depuis longtemps déjà et même dans les mines) au cours de la guerre de 14-18, par exemple.

On voit ci-dessous l'exemple de deux manuels scolaires de lecture des années cinquante qui permettent d'identifier clairement ce programme caché d'éducation en acte.

Figure 1 - Image de manuels de lecture de 1952

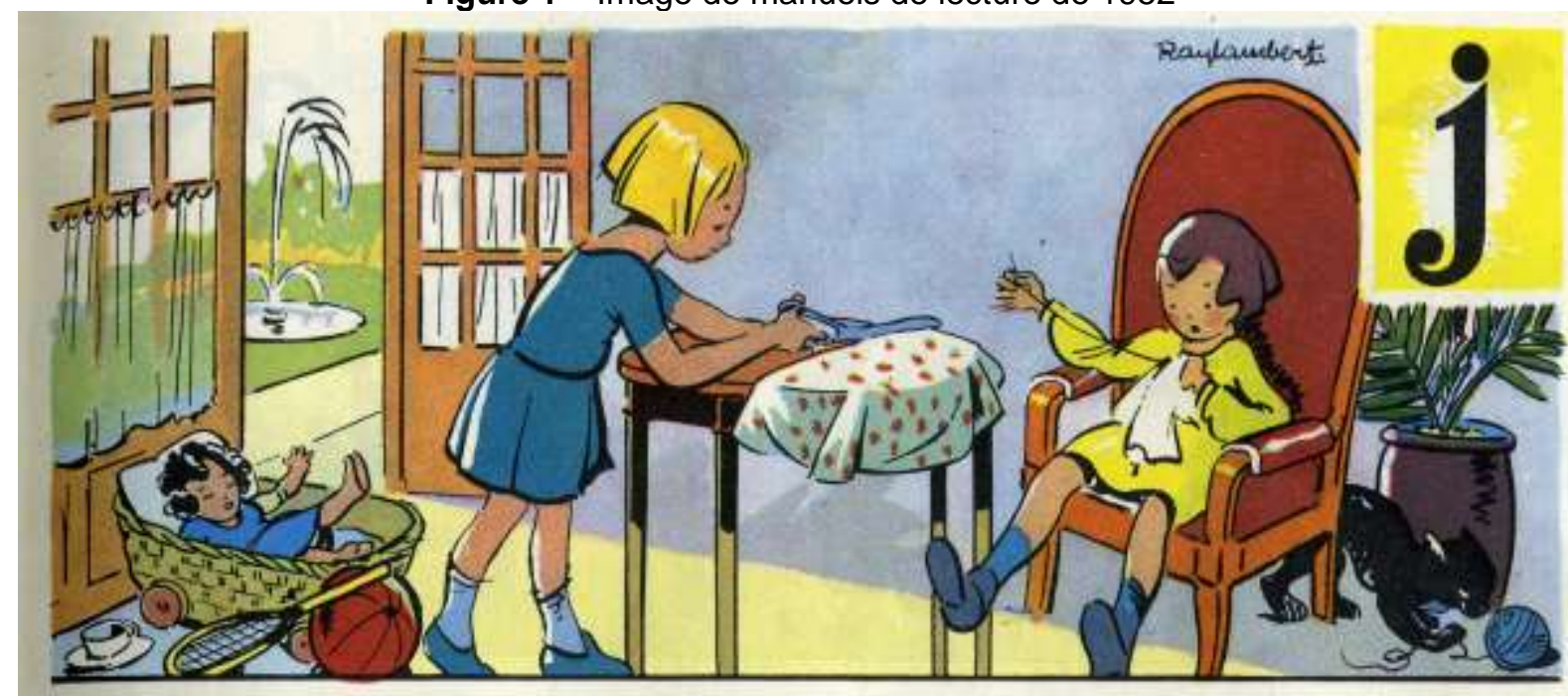

Source : Manuels de lecture (1952).

Figure 2 - Image de manuels de lecture de 1952

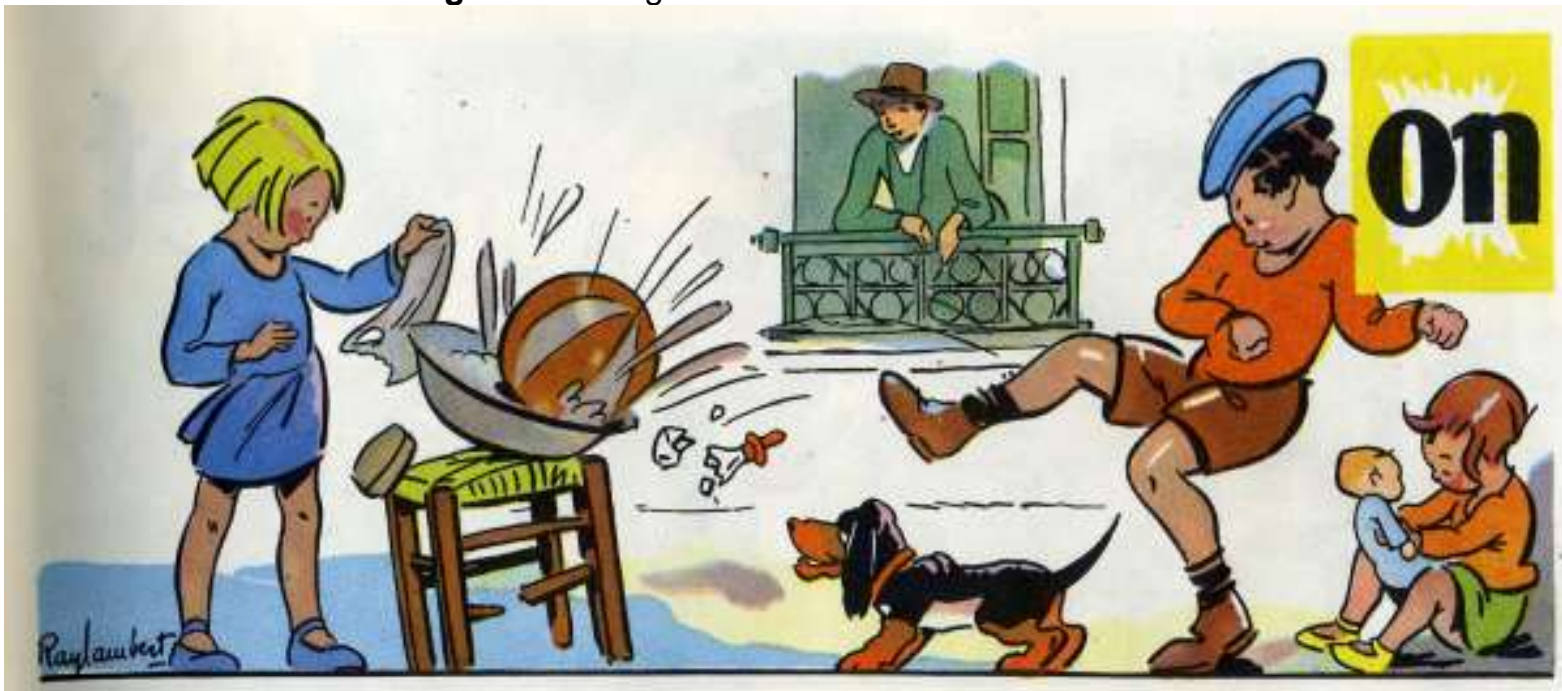

Source : Manuels de lecture (1952).

C'est sur cette base que l'on commencera à comprendre, en France, que la mixité n'est pas l'égalité. Le Journal of Gender Studies créé en 1991 initiera la mise en évidence d'éléments plus ténus et plus discrets, dans les processus éducatifs qui conduisent à un traitement inégalitaire des filles et des garçons. 
II y a quelques années une étude minutieuse (DORIGNY, 2009) des logiciels éducatifs présents au centre de documentation de l'ex-Institut Universitaire de Formation des Maîtres de Montpellier montrait que ceux-ci étaient traversés par d'innombrables stéréotypes de genre. Sur 13 logiciels éducatifs 11 avaient pour héros un garçon, 1 un robot et 1 une fille mais de multiples autres traitements inégalitaires des garçons et des filles ont été repérés dans l'école et dans l'enseignement au cours du temps.

C'est ainsi que Nicole Mosconi, récemment décédée, publiera, à la fin des années quatre-vingt, un ouvrage intitulé La Mixité dans l'enseignement secondaire : un faux-semblant (1989). Elle y montre que l'enseignement secondaire repose sur les bases d'un « masculin-neutre ». Autrement dit même si la mixité donne l'apparence de l'égalité ou a minima de la neutralité, les garçons ou les hommes dominent l'espace scolaires sous différentes formes. Les élèves garçons interagissent plus avec les enseignant(e)s, ils sont plus souvent sollicités par eux. Ils occupent au plan sonore et au plan spatial l'espace scolaire et le dominent. Ils prennent plus fréquemment la parole sans avoir été sollicités. A tel point que cette auteure pourra décrire une «loi des $2 / 3,1 / 3$ ». Celle-ci montre que dans une classe paritaire que ce soit une femme ou un homme qui enseigne, les garçons ont $2 / 3$ d'interaction avec lui et les filles seulement $1 / 3$. Mais lorsque I'on entrera plus tard dans le détail des interactions on va pouvoir constater à la suite de ces travaux princeps que d'autres programmes cachés d'éducation sont mis en œuvre par les enseignants à leur insu ou de manière relativement inconsciente.

\section{La multiplication des travaux sur le sujet}

Les travaux ultérieurs de Nicole Mosconi (2009) mais aussi d'autres chercheuses ou chercheurs comme Marie Duru-Bellat $(2004,2005)$; Thierry Terret, Geneviève Cogerino et Isabelle Rogowki (2006), sur l'EPS ; Isabelle Plante (2010) au Canada, sur les stéréotypes concernant les mathématiques et le français, de Julie Thomas (2010, 2013, 2018) sur l'activité physique dans les formations professionnelles ; de Stéphanie Rubi (2009), Corinne de Boissieu (2009), Cendrine Marro et Isabelle Collet (2009), dans la revue Recherches \& Educations: toutes révèlent la fécondité de la recherche sur le genre et l'éducation. 
Em outre, le rapport du Commissariat Général à la stratégie et à la prospective, publié en Janvier 2014, où écrivent Marie-Cécile Naves, Vanessa Wisnia-Weill, Marine Boisson-Cohen, Frédéric Lainé, Sylvie Octobre, Mathilde Reynaudi, Sarah Sauneron, Mona Zegaï ; les études récentes de Karine Isabelle (2010) sur la maternelle, Séverine Depoilly (2014), Gaïd Le Maner-Idrissi, Laëtitia Renault (2006); Annette Jarlegan (2009) ; Paul Fontayne (2002) ; Eveline Daréoux (2007) ; Amélie Seidah (2004) ; etc., montrent de manière fine; précise et surtout objectivée comment de multiples programmes cachés d'éducation sont mis en œuvre dès la petite section de maternelle, qui conditionnent des comportement différenciés des élèves garçons et des élèves filles. Evelyne Daréoux parle d'ailleurs de " la fabrication d'élèves filles et d'élèves garçons » tout autant que Corinne de Boissieu.

A titre d'exemple, parmi de nombreux autres programmes cachés d'éducation et pour ne pas lasser le lecteur, on peut constater que dans un cours de mathématiques l'enseignant pose des questions plus complexes aux garçons qu'aux filles (LEGRAND, 2016). II fait appel à la mémoire des filles et à la compréhension des garçons. II laisse, statistiquement, plus de temps aux garçons pour répondre qu'aux filles.

D'une manière plus générale, les enseignantes et enseignants font plus de remarques d'ordre disciplinaire mais aussi cognitives aux garçons qu'aux filles.

En Education Physique, les enseignants qui constituent des groupes de niveau en volley-ball, font en général un groupe fort constitué essentiellement de garçons et un groupe faible composé essentiellement de filles mais il est surtout intéressant d'étudier le groupe moyen qui, lui, est le plus souvent paritaire. Dans cette situation les enseignants et enseignantes font plus de remarques concernant le service, la réception et la passe aux filles et davantage de remarques concernant l'attaque et le contre aux garçons (TERRET ; COGERINO ; ROGOWSKI, 2006).

Dans l'ensemble des disciplines scolaires, lorsque l'on demande aux enseignants d'établir des relations égales avec les garçons et les filles, on se rend compte qu'ils ont tout de même plus d'interactions avec les garçons qu'avec les filles. Et dans cette situation si l'on demande leur ressenti aux élèves, les garçons se plaignent d'être délaissés (DUTEIL-DEYRIES, 2020).

On peut encore décrire le fait que les professeurs des écoles (GLEYSE, 2020) entre 5 et 8 ans croient à la supériorité des garçons en mathématiques et à celle des 
filles en littérature (ce qui est vrai pour ces dernières), alors qu'en réalité à cet âge les études ne montrent aucune différence de performance en mathématiques des filles et des garçons. Cette différence se constate seulement plus tard. On pense donc que cette croyance des enseignants agit comme une prophétie auto-réalisatrice (voir à ce sujet : Pygmalion at school, ROSENTHAL ; JACOBSON, 1966).

En fait, les exemples pourraient être multipliés à l'infini en passant de l'usage des coins jeux à la maternelle (souvent très stéréotypés), jusqu'aux actions des enseignants à tous les niveaux de classe visant à valoriser les garçons et à dévaloriser les filles ou du moins à induire certains types de comportements pour les garçons et d'autres types pour les filles.

Même en ce qui concerne les punitions Sylvie Ayral (2011) a très bien montré ainsi que Sophie Duteil-Deyries $(2014,2020)$ que les filles et les garçons ne sont pas punis de la même manière ni pour les mêmes types de fautes. Une fille est souvent plus sévèrement punie qu'un garçon dans les mêmes conditions. Les enseignants d'ailleurs ne sont pas du tout égalitaires dans leurs punitions. Selon l'étude réalisée par Sophie Duteil si des enseignants déclarent être égalitaires dans leurs punitions, lorsqu'on leur demande de vérifier objectivement s'ils le sont ils constatent sur une durée de deux semaines qu'ils ne le sont pas du tout. Les archives d'un établissement scolaire montrent la surreprésentation des garçons dans les élèves punis.

Bref, de multiples programmes cachés d'éducation sont mis en œuvre qui visent à fabriquer une élève fille et un élève garçon et non à mettre en place un traitement égalitaire des filles et des garçons à l'école. Cela, évidemment, a ensuite des conséquences importantes en terme de professionnalisation et à l'âge adulte.

\section{Conclusion}

Accroître les degrés de liberté des élèves filles et garçons dans l'institution scolaire en outillant le regard des acteurs, en les formant, tel est le but des travaux sur l'égalité filles-garçons en éducation et spécifiquement sur les programmes cachés d'éducation et sur les stéréotypes de genre à l'école. II s'agit d'ouvrir davantage le champ des possibles pour chaque élève en lui permettant ne pas se satisfaire d'une position assignée par l'histoire, la mémoire, les traditions, la société et la culture en général. 
Les recherches mises en œuvre aujourd'hui de manière relativement systématisée sur ces questions permettent d'entrevoir, chaque jour davantage, des pratiques presque invisibles, discrètes, occultées ou du moins difficilement perceptibles pour un non initié, mises en œuvre, entre autres, par les enseignants et les personnels d'encadrement, qui font que l'ont traite de manière non égalitaire les élèves filles et garçons.

Des recherches montrent que c'est dans la famille que s'initie ce processus mais que dès la moyenne section de maternelle et donc dès le développement de l'identité sexuée des élèves, l'école ne forme plus un individu élève ou une personne élève, mais bien un élève fille et un élève garçon ou plus clairement un élève-mâle porteur de stéréotypes masculins et une élève-femelle porteuse de stéréotypes féminins. Ces stéréotypes sont généralement hérité d'un passé patriarcal et conduisent à des inégalités en droits, devoir, dignité des filles et des garçons à l'école. Ce processus inégalitaire crée ensuite des situations de discrimination et surtout des difficultés d'accès à un certain nombre de domaines pour les femmes et à l'inverse une difficulté d'acceptation de certaines professions par les hommes mais aussi des comportements violents et/ou dangereux. Le champ des possibles est limité et restreint par les conditionnements infantiles scolaires, pesants. Probablement sont-ils pour partie à l'origine d'un certain nombre de comportements et de traitements dégradants pour les femmes et l'inverse de certaines positions machistes pour les hommes.

La prise de conscience dans le domaine de l'enseignement et de l'école de ces stéréotypes et de ces programmes cachés d'éducation, conduit à mettre en œuvre des programmes de recherche dont sont issus souvent de nouvelles formations qui porteront probablement leurs fruits à moyen termes.

\section{Références}

AYRAL, S. La fabrique des garçons : sanctions et genre au collège. Paris : Universitaires de France, 2011.

BELLOTTI, E. G. Du côté des petites filles. Paris : Des Femmes, 1974.

BEM, S. The measurement of psychological androgyny. Journal of consulting and Clinical Psychology, v. 42, n. 2, p. 155-162, 1974. 
BOISSIEU, C. Sexes et genres à l'école maternelle. Un essai de modélisation du concept de « genre scolaire ». Recherches \& Education, v. 2, p. 23-43, 2009.

BOURDIEU, P. La Domination masculine. Paris : Liber, 1999.

BUSCATTO, M. ; MARRY, C. Le plafond de verre dans tous ses éclats. La féminisation des professions supérieures au xx ${ }^{\mathrm{e}}$ siècle. Sociologie du Travail, v. 51, n. 2, p. 170-182, 2004.

CAMPONAR, G. C. et al. "Você não... nessa posição sim ou sim, um homem" : representações em torno do gênero e intervenção docente na formação, Educação \& Formação, Fortaleza, v. 5, n. 2, 2020.

COLLECTIF. Rapport du Commissariat Général à la stratégie et à la prospective. Paris, Gouv, 2014.

DAREOUX, E. Les stéréotypes de genre omniprésents dans l'éducation des enfants. Empan, v. 65, n. 1, p. 89-95, 2007.

DEPOILLY, S. Filles et garçons au lycée pro. Rapport à l'école et rapport de genre. Rennes : PUR, 2014.

DORIGNY, A. Les logiciels éducatifs et les stéréotypes de genre. Mémoire Master Sciences de l'Education, Faculté d'Education : Montpellier, 2009.

DURU-BELLAT, M. L'Ecole des filles : Quelle formation pour quels rôles sociaux ?. Paris : L'harmattan, 2004.

DUTEIL-DEYRIES, S. " Les filles sont des pipelettes les garçons des bagarreurs ». Une étude des stéréotypes de genre concernant les punitions et les sanctions dans un établissement scolaire. Mémoire de master 2. Montpellier : ESPE, 2013.

DUTEIL-DEYRIES, S. Transgression scolaire au prisme du genre. De l'invisibilité des filles à la survisibilité des garçons. Paris : L'Harmattan, 2020.

FONTAYNE, P.; SARRAZIN, P.; FAMOSE, J.-P. Effet du genre sur le choix et le rejet des activités physiques et sportives en éducation physique et sportive : une approche additive et différentielle du modèle de l'androgyne. Movement and Sport Sciences, n. 45, p. 4566, 2002.

GLEYSE, J. Le Genre de l'école en France. De la mixité à l'inégalité occultée. Expérimentations et propositions de transformations. Paris : L'Harmattan, 2020.

GLEYSE, J.; GARCIA, C.; CANAL, J.-L. Image of women in Literature on Physical Education and Sport : Representations of Social Transformation in France under the Thrid Republic, 1870-1939. Sport, Education and Society, v. 9, n. 3, p. 363-381, 2004.

GLEYSE, J.; SOARES, C. L. Os manuais escolares franceses de Educação Física, de Hygiene e de Moral entre 1880-2004 seriam sexistas? Educaçao \& Sociedade, Campinas, v. 28, n. 102, p.137-159, 2008. 
ISABELLE, K. Pourquoi la mixité dans les classes ne provoque-t-elle pas spontanément l'égalité de traitement entre les filles et les garçons?. Tréma, v. 32, p. 49-67, 2010.

JARLEGAN, A. Genre et éducation. De l'intérêt de la prise en compte du genre en éducation. Recherches \& Education, v. 2, p. 11-21, 2009.

LEGRAND, C. Les Stéréotypes de genre en Mathématiques et leur influence sur les résulats, la motivation et le cheminement scolaire des élèves. Mémoire M2. ESPE : Angers, 2016.

LE MANER IDRISSI, G.; RENAULT, L. Développement du « schéma de genre » : une asymétrie entre filles et garçons. Enfance, v. 58, n. 3, p. 251-265, 2006.

MARRO, C.; COLLET, I. Les relations entre les filles et les garçons en classe. Qu'en disent-elles ? Qu'en disent-ils ?. Recherches \& Education, v. 2, p. 45-71, 2009.

MOSCONI, N. La Mixité dans l'enseignement secondaire : un faux-semblant. Paris : PUF, 1989.

PLANTE, I. Adaptation et validation d'instrument de mesure des stéréotypes de genre en mathématiques et en français. Mesure et Evaluation en Education, v. 33, n. 2, p. 1-34, 2010.

PROST, A. Histoire de l'enseignement en France. 1800-1967. Paris : PUF, 1968.

PROST, A. Éducation, société et politiques : une histoire des enseignements en France de 1945 à nos jours. Paris : Seuil, 1992.

RODRIGUEZ, N. B. Notas para problematizar la ética de sí en la educación del cuerpo : la clase de Educación Física como territorio de disputas de género. Educação \& Formação, Fortaleza, v. 5, n. 2, 2020.

ROSENTHAL, R.; JACOBSON, L. Pygmalion in the classroom. New-York : Holt, Rinehart \& Winston, 1966.

RUBI, S. Contextualisation des différences sexuées et genrées du sentiment de violence déclaré par des élèves scolarisés populaires. Recherches \& Education, v. 2, p. 93-119, 2009.

SEIDAH, A. Perception de soi à l'adolescence : différences entre filles et garçons. Enfance, v. 56, n. 4, p. 405-420, 2004.

SOUZA RIOS, P. P. ; DE MELO CARDOSO, H. ; FERREIRA DIAS, A. Concepções de gênero e sexualidade d@s docentes do curso de licenciatura em Pedagogia: por um curriculo Queer. Educação \& Formação, Fortaleza, v. 3, n. 2, 2018.

TERRET, T. ; COGERINO, G.; RIGOWSKI, I. Pratique et représentations de la mixité en EPS. Paris : EPS, 2006. 
THOMAS, J. Etre une fille et s'engager dans une filière scolaire de garçons : la place des activités physiques et sportives dans la construction de l'« atypicité » scolaire. Thèse, Paris 11, dir. C. Louveau, 2010.

THOMAS, J. Le corps des filles à l'épreuve des filières scolaires masculines. Le rôle des socialisations primaires et des contextes scolaires dans la manière de faire « faire le genre ». Sociétés contemporaines, Paris, v. 2, n. 90, 2013.

WILLIAMS, J. E. ; BENNETT, S. M. The définition of Sex Stereotypes via the Adjective Check list. Sex Roles, v. 4, n. 1, p. 327-337, 1975.

WILLIAMS, J. E. ; SATTERWHITE, R. C. ; BEST, D. L. Pancultural gender stereotypes revisited : the five factor model. Sex Roles, v. 40, p. 513-525, 1999.

\footnotetext{
Jacques Gleyse, Université de Montpellier

i- https://orcid.org/0000-0002-8459-5958

Docteur en sciences de l'éducation - Université de Paris X, Nanterre. Professeur des Universités à I'IUFM de Montpellier, Université de Montpellier 2 (Montpellier - Hérault - France).

Contribution d'auteur: Paternité complète de l'article.

E-mail: jacques.gleyse@orange.fr
}

Éditeur responsable: Lia Machado Fiuza Fialho Experts ad hoc: Inmaculada Díaz et Celia Pérez

\section{Comment citer cet article (ABNT):}

GLEYSE, Jacques. Stéréotypes de genre et programmes cachés d'éducation dans l'enseignement en France. Une revue de littérature sur les inégalités occultées. Educ. Form., Fortaleza, v. 6, n. 2, e4625, 2021. Disponible sur: https://revistas.uece.br/index.php/redufor/article/view/4625

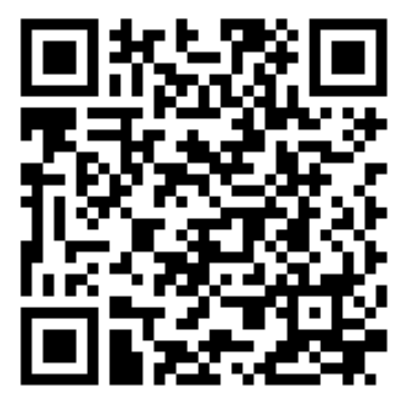

Reçu le 16 janvier 2021.

Accepté le 18 février 2021.

Publié le 2 avril 2021. 